\title{
TRAMAS Y LABERINTOS: SOCIOLOGÍA E IDENTIDAD CULTURAL LATINOAMERICANA ${ }^{1}$
}

\author{
WEAVINGS AND LABYRINTHS: LATIN AMERICAN \\ SOCIOLOGY AND CULTURAL IDENTITY
}

\section{JORGE IVÁN VERGARA², JORGE VERGARA ESTÉVEZ ${ }^{3}$ Y HANS GUNDERMANN ${ }^{4}$}

\section{RESUMEN}

En el artículo se realiza una crítica de las principales interpretaciones sobre la identidad cultural latinoamericana, mostrando sus mayores dificultades conceptuales y teóricas. Se concluye señalando algunas propuestas para reenfocar el tema desde una perspectiva sociológica más compleja y actual.

Palabras clave: América Latina, identidad cultural, interpretaciones, crítica sociológica.

\section{ABSTRACT}

This paper is a critique of the main interpretations of Latin American cultural identity, examining major conceptual and theoretical difficulties. It concludes by presenting some proposals to refocus the topic by using a more current and complex sociological perspective.

Keywords: Latin America, cultural identity, interpretations, sociological critique.

Recibido: 05.09.11. Aceptado: 09.03.12.

${ }^{1}$ Este artículo fue elaborado en el marco del proyecto Fondecyt 1085324 (2008-2010), a cargo de Jorge Iván Vergara y Hans Gundermann.

${ }^{2}$ Instituto de Investigaciones Antropológicas, Universidad de Antofagasta. Antofagasta, Chile. E-mail: jorgeivan.vergara@uantof.cl

${ }^{3}$ Departamento de Educación, Facultad de Filosofía y Humanidades, Universidad de Chile. Santiago, Chile. E-mail: vergaraestevez@gmail.com

${ }^{4}$ Instituto de Investigaciones Arqueológicas y Museo Rvdo. Padre Gustavo Le Paige, Universidad Católica del Norte. San Pedro de Atacama, Chile. E-mail: hgunder@ucn.cl 


\section{INTRODUCCIÓN}

E L OBJETIVO DE ESTE ARTíCULO es hacer una crítica sociológica de las principales interpretaciones acerca de la identidad cultural latinoamericana. Se trata de abordar la rica pero problemática reflexión sobre nuestra identidad, la que se asemeja a un laberinto conceptual: cada interpretación suscita problemas conceptuales que no puede resolver $y$, al mismo tiempo, pretende, en buena medida, haber agotado la discusión. Por ello, y confrontadas entre sí, las distintas tesis representan otras tantas alternativas excluyentes pero igualmente unilaterales. La nuestra pretende ser una contribución a la conformación de una trama interpretativa más coherente, compleja y plural. Con este fin, contextualizaremos brevemente la discusión sobre la cuestión identitaria en la Región, proponiendo algunos lineamentos para su análisis desde la sociología; describiremos a continuación las tesis clásicas sobre la identidad latinoamericana para hacer, después, una crítica comparativa de ellas y, finalmente, exponer algunas conclusiones sobre el tema.

Partimos de la constatación de que actualmente nos enfrentamos a una dinámica compleja y contradictoria, en la que conviven el debilitamiento de las macro-identidades, sobre todo las de clase y nacionales; la emergencia y difusión de movimientos culturales heterogéneos cuyo flujo globalizado se posibilita con las nuevas tecnologías de la información; el crecimiento de las identidades ligadas a espacios micro-sociales, visible en el fenómeno de las "tribus urbanas" y de grupos locales de diverso tipo; y el resurgimiento de las identidades étnicas y regionales.

Para Castells este auge de las micro-identidades sería una reacción defensiva ante la globalización cultural liderada por los medios de comunicación masivos (mass media) y el retroceso del Estado-nación y su monocultura. Los sectores abandonados por la globalización encontrarían en ellas "principios alternativos de sentido y legitimidad" al "principio de la ciudadanía" en el cual se fundaba la integración social (Castells, 2006: 15-24). Sin embargo, la relación entre los dos fenómenos no es necesariamente de mutua exclusión. Dichos movimientos participan de tendencias generales que la globalización misma ha favorecido (o, al menos, no excluido) como es el caso de las denominadas "tribus urbanas": hip-hoperos, rastafari y otros (Maffesoli, 2004). En cierta medida, éstos existen gracias a los medios de comunicación de masas, pero pretenden encarnar principios alternativos de convivencia social. En vez de considerarlos como opuestos a la globalización, habría que precisar los ejes de oposición y afirmación específicos. 
Asimismo, podría estudiarse sus relaciones con procesos de diferenciación social al interior de distintos segmentos etarios, de clase y de género, o sea, con identidades sociales tradicionales y más inclusivas. Esta diferenciación operaría, en primer lugar, en términos de inclusión/exclusión respecto a las posibilidades de acceso al consumo ofrecidas por la globalización (Bauman, 1999: 103-133).

Por su parte, Bauman ha afirmado que estas nuevas identidades y los movimientos sociales conformados en torno a ellas se caracterizan por su fragilidad temporal (confinando a los individuos a vivir en un presente continuo, sin pasado ni futuro), y la dificultad de estructurar relaciones estables (2004: 60-72). Sin embargo, como ha sostenido Michel Maffesoli, esta misma condición permite al individuo asumir distintos roles en las diferentes "tribus efectivas" en que participa, conjugando un intenso sentido de comunidad con una afirmación fuerte de la individualidad (Maffesoli, 2004).

Distinto sería el caso de la emergencia de las identidades colectivas de viejo cuño, como las identidades étnicas, sin que ello suponga necesariamente continuidad cultural con las tradiciones culturales del pasado. Por el contrario, la afirmación étnica en Latinoamérica se da en un contexto de cambios profundos en las poblaciones indígenas, a la vez que ellas participan de una tendencia mundial en pro del reconocimiento de sus derechos y utilizan los recursos mediáticos y tecnológicos provistos por la globalización.

Todo lo anterior conlleva la generación de una situación nueva y distinta a la de 20 ó 30 años atrás y, hasta cierto punto, una incertidumbre respecto de nuestra pertenencia cultural. En consecuencia, una primera proposición a resaltar es que la sociología (y las ciencias sociales, en general) no pueden tratar el tema de la identidad como un fenómeno ligado sólo a la conservación de ciertas formas de vida social, pues la identidad misma está sujeta a cambios que forman parte de transformaciones sociales más amplias.

En segundo lugar, la sociología latinoamericana debe ser capaz de mostrar la compleja dinámica de los procesos de cambio social, apartándose de una mirada lineal respecto a la modernización como sinónimo de progreso, mostrando también sus limitaciones y consecuencias negativas.

Tercero, la sociología latinoamericana debe ser capaz de renovarse teórica y metodológicamente para responder a las exigencias que plantea un análisis complejo del fenómeno de las identidades culturales en relación con América Latina. Después de haber constituido un importante foco de atención sociológica latinoamericana y mundial, la cuestión identitaria ha 
pasado a un segundo lugar, en buena medida debido a los escasos avances que se dieron en la discusión. Nuestro trabajo quiere ser un aporte en su revitalización desde una mirada sociológica amplia y en diálogo con otras ciencias sociales, con las humanidades y con la filosofía.

\section{EL CONCEPTO DE IDENTIDAD CULTURAL EN RELACIÓN CON AMÉRICA LATINA}

El concepto de identidad cultural es uno de los más polisémicos y, a la vez, más recurrentes en las ciencias sociales contemporáneas, lo que obliga a abordar, aunque sea brevemente, la cuestión conceptual. La identidad se debe entender en plural. El problema de la identidad es el problema de las identidades; por ende, se debe considerar siempre la diferenciación entre identidades como un aspecto inseparable de su constitución, diferenciación que puede adquirir distintos signos (de oposición, de subordinación o de incorporación, los principales).

Se pueden distinguir tres enfoques al respecto. El primero ve la identidad cultural como algo estático, como una esencia que no cambia y que permite definir lo que es propio de un grupo o un pueblo. Esta es una postura esencialista ${ }^{5}$. Contrapuesto al anterior, existe también un enfoque histórico de las identidades, que afirma que son algo abierto, siempre en proceso de cambio nunca completo ${ }^{6}$. Una tercera aproximación teórica enfatiza el hecho de que la identidad es una construcción discursiva, una creación de carácter fundamentalmente narrativo o un conjunto de "posiciones de sujeto" (Foucault) en permanente cambio (Hall, 1992 y 1997).

Preguntarse por la identidad latinoamericana requiere situarse y relacionar el debate con estas perspectivas teóricas. Lo que predominó históricamente fue el esencialismo, mientras en los últimos tiempos han convivido interpretaciones históricas y post-modernas, las cuales enfatizan la construcción discursiva de la identidad cultural, aunque sus premisas difieran considerablemente.

Por otro lado, se plantea la pregunta de si tiene sentido hablar de una identidad cultural latinoamericana, ya que para algunas corrientes este se-

\footnotetext{
${ }^{5}$ Véase la interesante caracterización y crítica del esencialismo realizada por Stuart Hall (1990 y 1992).

${ }^{6}$ Los mejores representantes de esta posición en América Latina son Jorge Larraín (1996 y 2000) y Gilberto Jiménez (1993 y 1994).
} 
ría un seudo-problema. Una primera posición sostiene que las transformaciones de tipo económico y tecnológico han ido unificando los países, favoreciendo el surgimiento y desarrollo de identidades globales. Algunos, inclusive, afirman que vamos en camino hacia una cultura mundial en la cual subsistirían ciertas diferencias de lenguajes, costumbres, sensibilidades, pero con un carácter secundario. Desde esta perspectiva, no tendría sentido pensar en la identidad latinoamericana, porque ésta ha dejado ya o está dejando de existir como diferenciable dentro de dicha cultura.

Del mismo modo, para una postura postmoderna tampoco tiene sentido hablar de identidad latinoamericana. En sus versiones más radicales, rechaza la idea misma de identidad. Sólo existiría un conjunto de acontecimientos y experiencias en continuo cambio; el yo y la identidad serían una ilusión o ficción construida a partir de un relato. Una variante más moderada es la de García Canclini (1990 y 2000), para quien toda cultura o identidad, en cualquier región o lugar, está cruzada o constituida por un fenómeno de "hibridación", de pérdida de lo propio y de cruce con otras. En consecuencia, en el mejor de los casos, cabría preguntarse cuáles serían nuestras hibridaciones específicas, pero no suponer que haya una forma de valoración o de identidad propias de América Latina.

\section{TESIS CLÁSICAS SOBRE LA IDENTIDAD CULTURAL LATINOAMERICANA}

La primera gran interpretación es la indianista. Nuestra cultura ha sido indígena y sigue siéndolo en sus raíces ocultas. Todos los cambios o intentos de cambio de origen occidental no han logrado impregnar ni transformar la realidad profunda de nuestro ser. Debemos volver a nuestras raíces ocultas bajo el manto ajeno impuesto por los dominadores extranjeros.

Fausto Reynaga puede ser considerado el padre fundador de esta corriente, cuyas ideas fueron continuadas por su hijo Ramiro. En una de sus principales obras, Ramiro Reynaga sostiene la necesidad de emprender una descolonización de los pueblos andinos similar a la que tuvo lugar en Africa y en otros lugares del mundo. Éstos deben liberarse de la quintocentenaria opresión cultural de España y de sus herederos, las repúblicas criollas, que no han logrado acabar con la civilización andina que floreció en el Tawantinsuyu, que se caracterizó por "la igualdad o democracia sideral [que] se refleja en la igualdad de la comunidad" (Reynaga, 1981 [1978]: 17). En ella no existieron el hambre, ni la explotación ni la guerra, "aje-

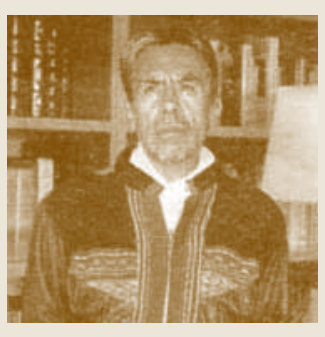

R. Reynaga 


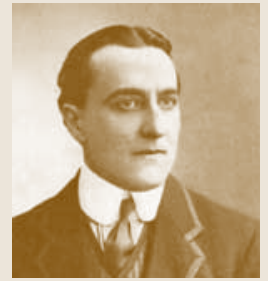

R. de Maetzu

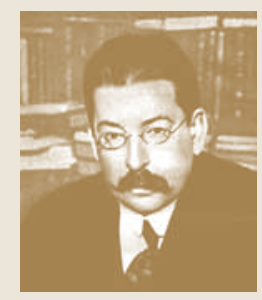

E. Rodó

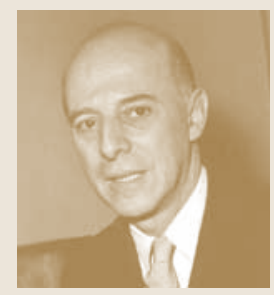

J. Eyzaguirre

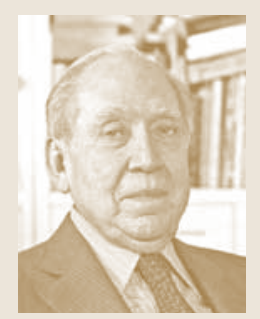

U. Pietri na a la armonía cósmica y, por ende, a la sabiduría inca" (Ibíd: 25). Pero la influencia de Occidente ha producido la desindianización de muchos quechua-aymaras, proceso que debe ser contrarrestado completamente, recuperando sus lenguas, vestimentas propias y toda su organización originaria (Ibíd: 269-297).

El hispanismo sostiene una versión completamente opuesta de la identidad latinoamericana a la del indianismo. Su origen está en el pensamiento conservador español de fines del siglo XIX, de Ramiro de Maeztu y otros, quienes sostuvieron la idea de la originalidad, excepcionalidad y superioridad de la cultura hispana -especialmente la de la época imperial, de "los siglos de oro"- respecto a la europea y especialmente la anglosajona. Estos autores influyeron decisivamente en los hispanistas latinoamericanos: José Enrique Rodó, Osvaldo Lira y Jaime Eyzaguirre, entre los más conocidos.

Tomando como referencia las ideas de Eyzaguirre, se puede reconstruir muy sintéticamente el planteamiento hispanista sobre nuestra identidad. Sostiene que somos hispanos o herederos de la tradición española. El aporte indígena ha sido menor $y$, en todo caso, sus "valores esenciales" han sido incorporados al patrimonio hispanoamericano (Eyzaguirre, 1961). Nuestra unidad histórica y cultural ha sido aportada por la cultura hispana y sus valores fundamentales: el idealismo, el honor, el respeto a la autoridad y el sentido religioso católico. Pese a todos los intentos de los estados posteriores a la Independencia para borrar nuestra tradición cultural seguimos siendo inherentemente hispánicos, o iberoamericanos, y la única manera de superar la crisis cultural presente es recuperar esta tradición (Eyzaguirre, 1946).

La tesis occidentalista, por su parte, intenta superar el dilema anterior planteando que nuestra identidad latinoamericana consiste en que somos occidentales o estamos en vías de serlo. A diferencia de Asia y África, América Latina no presenta culturas tradicionales vigentes que sean realmente distintas a la occidental. Para el destacado politólogo francés Alain Rouquié, América Latina es "el extremo occidente" (Rouquié, 1997 [1984]). Similar es la postura de Uslar Pietri, quien sigue a Paz en la idea de que somos "un polo de Occidente", pero "evidentemente excéntrico" (Pietri, 1991: 147).

Esta interpretación tiene su origen histórico a mediados del siglo XIX, con los representantes del liberalismo-positivista: Juan Bautista Alberdi, Francisco de Miranda y Domingo Faustino Sarmiento. Todos ellos sostuvieron la necesidad de occidentalizar a América Latina, contra la tradición hispana católica y la cultura indígena que, en muchos casos, aparecían uni- 
das o como una sola, que expresaban la "barbarie" frente a la necesidad de desarrollar "la civilización", como decía Sarmiento.

Actualmente, los intérpretes más característicos de esta posición serían los partidarios de la modernización neoliberal. José Joaquín Brünner defiende la existencia de "una identidad moderna de América Latina" o, mejor, de identidades "proporcionadas por los medios de comunicación, en particular la televisión, y por los múltiples otros lenguajes que se generan con la vida urbana, con los movimientos del mercado cultural, y con las nuevas formas de inserción de los países en la economía del mundo" (Brünner, 1993: 211). En consecuencia, dichas identidades coinciden en buena medida con las que proporciona el mercado y desplazarían a las tradicionales: indígena, campesina, ibérica, nacional y otras.

La cuarta tesis es la del mestizaje. Tiene su origen más antiguo en Bolívar y su primera versión la elaboró José Vasconcelos, con su concepto y utopía de "la raza cósmica". Por su gran difusión y la diversidad de versiones que existen de ella, la tesis del mestizaje puede considerarse la más importante de las interpretaciones clásicas sobre la identidad latinoamericana. Sostiene que existe en América Latina una cultura propia, que es el resultado de la fusión de las culturas originarias, negra y europea. Pedro Morandé, uno de sus principales autores, la denomina "síntesis cultural mestiza". Ésta constituiría inclusive una modernidad propia y distinta a la "modernidad ilustrada": la "modernidad barroca", con un fuerte acento religioso y ritual; por ende, en las imágenes o símbolos y en la legitimación cúltica y no monetaria del trabajo (Morandé, 1984: 149-187).

Se considera a Octavio Paz como un representante de esta corriente. Sin embargo, una lectura más cuidadosa demuestra que no participa de la tesis del mestizaje, sino que tiene su propia interpretación. La identidad mexicana ( $y$, en buena medida, también la latinoamericana) es problemática y tensionada. En consecuencia, no hay una cultura que integre los elementos que siguen existiendo, siempre en conflicto, como lo plantea en su obra más conocida, El laberinto de la soledad (Paz, 2004a [1959]). Paz sostiene allí que "el mexicano no quiere ser indio ni español. Tampoco quiere descender de ellos. Y no se afirma como mestizo sino como abstracción: es un hombre. Se vuelve hijo de la nada. Él empieza de sí mismo" (Paz, 2004a [1959]: 79). Podría leerse esto como una invitación a crear una nueva identidad, más allá de las tradiciones, pero el análisis del libro muestra que, para Paz, México, y quizás toda América Latina, tienen una vocación de modernidad que no pueden alcanzar. Por eso se puede afirmar que la identidad mexicana es un caso de conciencia desgraciada: quiere alcanzar la modernidad,

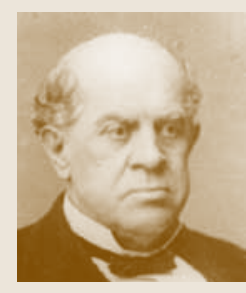

A. Bello

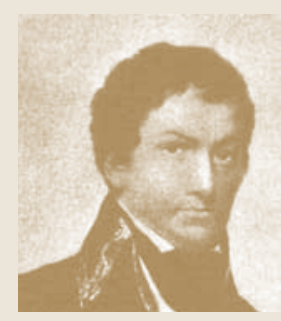

F. de Miranda

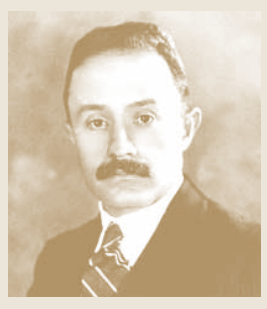

J. Vasconcelos

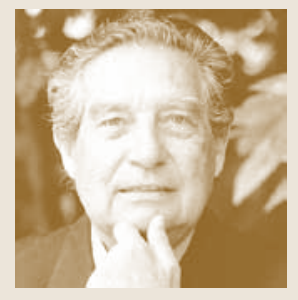

O. Paz 
pero está presa, de una parte, de un pasado indígena azteca y, de otro, de una tradición colonial que no se lo permiten. La masacre de Tlatelolco es un momento significativo, un nódulo histórico, que muestra que el peso del pasado impide la construcción de un presente posible y deseable ( $\mathrm{Paz}$, 2004b [1970]). De esta manera, los mexicanos están presos en un laberinto de la identidad. Es decir, no pueden construir una identidad armónica e integrada, porque sus distintas tradiciones son disímiles y, en todo caso, aunque fueran cercanas entre sí, no les permiten acceder a la modernidad a la cual aspiran y a la que no pueden, tampoco, renunciar.

\section{ANÁLISIS CRÍTICO DE LAS TESIS E IMPLICACIONES PARA EL DEBATE}

Las cinco tesis expuestas muestran, pese a sus diferencias, análogos y graves problemas. En primer lugar, ninguna responde satisfactoriamente a la pregunta sobre la identidad latinoamericana. En todas encontramos supuestos teóricos cuestionables. Uno de ellos es el concepto de identidad cultural desde el cual han sido pensadas. Se diría que hay una cierta tradición intelectual: estas tesis se fueron formulando y se siguen desarrollando a partir de una ingenuidad conceptual. "Identidad" es un concepto extremadamente complejo, sobre el cual hay muchas teorías y versiones pero, en general, hasta los años ochenta, los autores latinoamericanos no estaban al tanto del debate sobre dicho concepto ni habían participado en él. En sus interpretaciones, el concepto de identidad aparece como un supuesto no problematizado, una actitud intelectual inadecuada para construir interpretaciones complejas. Si no se hace una reflexión sobre él, la investigación carece de un norte claro, de autoconciencia reflexiva, y ese es uno de los problemas más serios que encontramos en la literatura sobre el tema, situación que cambia en la década siguiente. En efecto, los últimos años se constata en América Latina un reconocimiento de dicha complejidad conceptual y el desarrollo de diversos enfoques relativamente originales sobre la identidad cultural, lo que ha enriquecido el debate teórico general.

Asimismo, e independientemente de los contenidos o rasgos que se atribuyan a la identidad nacional o latinoamericana, en las tesis analizadas predomina la idea de que se trata de una unidad de lo diverso o es una propiedad determinada, algo ya alcanzado o realizado. Esta asimilación conceptual de la identidad con tradiciones culturales que se supone definitivas casi siempre se asocia con la concepción esencialista descrita antes. 
Se observa también una fuerte tendencia a homologar o considerar que existe una identidad cultural latinoamericana y asimilar a ella las identidades nacionales. No se establecen demasiadas diferencias en la manera de concebir una y otra, en contraste con Europa, donde las identidades nacionales, e incluso étnicas y regionales son mucho más definidas y arraigadas que la identidad europea. Esto podría deberse al hecho de que nuestras culturas nacionales, pese a sus indudables diferencias, poseen muchos rasgos compartidos, como han señalado diversos autores desde Bolívar hasta Felipe Herrera.

Pareciera que la tesis del mestizaje cultural es menos objetable en este respecto, pero los autores que la sostienen no han problematizado suficientemente la propia noción de mestizaje ${ }^{7}$."Mestizaje cultural" es una metáfora, más que un concepto, usado para referirse a un fenómeno que no puede explicarse como consecuencia de la fusión biológica de distintos grupos étnicos, por ello requiere ser interpretado con categorías propias del análisis cultural. No se ha reflexionado sistemáticamente, tampoco, sobre el sello colonial del mestizaje cultural de los siglos XVI al XVIII ni sobre el carácter incompleto y conflictivo de los procesos de mestizaje cultural y de los procesos contrarios de aculturación, que buscan eliminar o minimizar formas culturales mestizas. Nadie puede negar la existencia de fenómenos de síntesis cultural en la religiosidad popular, el arte o la pintura, entre otros (Arguedas, 1981: 148-172 y Gisbert, 1999). El problema es si se puede decir que toda la cultura latinoamericana y cada una de las culturas nacionales han realizado este proceso de mestizaje. La respuesta es negativa: se confunde la parte con el todo, se generaliza a partir de algunos fenómenos, o se considera realizado históricamente lo que es sólo una tendencia de la realidad, allí muestra esta tesis toda su debilidad.

La propia experiencia e idea de mestizaje tampoco resuelve el problema; es una idea que, bajo su aparente unidad conceptual, encubre fenómenos hasta cierto punto heterogéneos y contradicciones internas no resueltas. Existen diversos tipos de mestizajes culturales. Es diferente el que se produce, en la Colonia, entre tradiciones indígenas y negras, como en el caso de los afro-bolivianos; que el que se da entre las tradiciones de habla alemana del siglo XIX y las del sur de Brasil, Argentina o centro-sur de Chile; difiere el sincretismo religioso de Chiloé de aquel de los Andes peruanos, etc.

\footnotetext{
${ }^{7}$ Una importante excepción es el artículo de Montecino (2005 [1997-1998]).
} 
Al mismo tiempo, las cuatro tesis mencionadas se mantienen y siguen teniendo una fuerte presencia en la experiencia cotidiana de la identidad, independientemente de sus debilidades teóricas. Nuestra hipótesis de trabajo es que corresponden a formas de vida, aspiraciones y proyectos de importantes sectores de las sociedades latinoamericanas (Vergara y Vergara, 1996). En ese sentido, tendrían vigencia social y cultural, puesto que poseen la capacidad de hacer comprensible o dar sentido a las prácticas sociales de grupos específicos.

Cada una de estas tesis alcanza mayor verosimilitud y arraigo en ciertos espacios culturales. La tesis indianista tiene, principalmente, presencia en vastos sectores del mundo andino, aunque también en Guatemala y ciertos sectores en México. Esto hace verosímil o convincente la idea de que la identidad indígena es la más fundamental de esas naciones o esa región. La tesis de la modernidad cultural es más plausible para los grupos blancos o de migrantes europeos de las grandes ciudades latinoamericanas, los cuales han perdido conexión con las culturas vernáculas y se identifican con la cultura "moderna". A su vez, la afirmación del mestizaje cultural mantiene presencia en sectores donde dichos fenómenos, por razones históricas, han sido más pronunciados o profundos. Igualmente, la visión hispanista retiene vigencia en sectores sociales tradicionales y en grupos católicos conservadores. Asimismo, hay sociedades latinoamericanas donde algunas de estas tesis guardan especial vigencia por la historia cultural de dichas sociedades. Así sucede, por ejemplo, en Bolivia, Perú o Guatemala con la tesis indianista, en Uruguay y Argentina con la de la modernidad, o en Colombia o México, con la del mestizaje.

En el análisis de las referidas tesis hay que considerar las diferencias significativas entre las sociedades latinoamericanas, que éstas tienden a ignorar, pretendiendo ser válidas para el conjunto de ellas. Por el contrario, hay que reconocer la gran heterogeneidad interna de nuestra Región y evitar, así, subsumir su diversidad efectiva en una única gran expresión cultural, por importante que ella sea. Al respecto, Pablo Guadarrama ha señalado: "En el actual creciente proceso de internacionalización de la vida social, en que los pueblos se conocen cada vez mejor, resulta progresivamente más fácil percatarse de las similitudes y diferencias que subsisten en las culturas de las diferentes regiones. Incluso en un mismo país en ocasiones se aprecia una diversidad tan grande de manifestaciones culturales que podría poner siempre en tela de juicio el concepto de identidad cultural" (Guadarrama, 1992: 120; también Cornejo-Polar, 1990). 
Uno de los mayores problemas que comparten las tesis mencionadas es, justamente, partir del supuesto de que existe una única identidad cultural latinoamericana. Las tesis difieren sobre cuál es su contenido, pero comparten este principio fundamental, que ya había sido cuestionado por Leopoldo Zea en un importante trabajo de 1983. Según este destacado pensador mexicano, en América Latina hay una presencia simultánea y conflictiva de componentes culturales muy diversos, que coexisten muchas veces en capas o conviven al mismo nivel, pero que no resulta de ello una sola identidad cultural, cualquiera sea ésta. Nuestra situación de heterogeneidad cultural nos abre la posibilidad de acceder a una modernidad donde lleguen a articularse nuestros propios y disímiles componentes: los más propios nuestros o más cercanos a nuestra cultura con la herencia de las culturas desarrolladas, etcétera. América Latina sería "una cultura surgida de la unión, pero no de asimilación de la cultura propia de estos hombres (de los pueblos autóctonos). Cultura de expresiones encontradas, y por serlo, lejos de mestizarse, de asimilarse, se han vuelto yuxtapuestas. Yuxtaposición de lo supuestamente superior sobre lo que se considera inferior, relación en que el mestizo, tanto cultural como racialmente se transforma en conflicto interno. Conflicto del hombre que lleva en su sangre y cultura al dominador y al bastardo" (Zea, 1983: 289).

En esta lectura, las identidades latinoamericanas serían internamente y externamente conflictivas, conviviendo en ellas dos posiciones y figuras sociales contrapuestas, las del dominador y la del dominado, que conviven en un mismo sujeto y colectividad.

\section{CONCLUSIONES}

Considerando los argumentos recién expuestos, resulta sorprendente que estas cinco tesis clásicas hayan coexistido por tanto tiempo, siendo tan opuestas entre sí. Tal como han sido formuladas, es obvio que no pueden ser todas válidas. Sin embargo, creemos que son parcialmente verdaderas pues cada una de ellas muestra, o patentiza, modalidades y formas de vida histórico-culturales que existen en América Latina. Lo que las hace incompletas, y en este sentido falsas, sería su pretensión de haber resuelto el problema de la identidad latinoamericana. Podría decirse, siguiendo a Hegel, que tendrían razón en lo que afirman, pero no en lo que niegan. Más aún, es discutible que una interpretación identitaria pueda ser "ver-

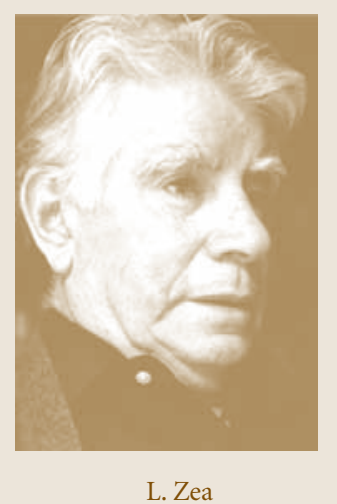

L. Zea 
dadera". Podemos preguntarnos si los enunciados mediante los cuales se expresa dicha interpretación son del mismo tipo que las proposiciones descriptivas, explicativas y comprensivas que constituyen las ciencias sociales empíricas, respecto de las cuales cabe decidir si son "verdaderas" o "falsas". Se asemejarían a los discursos estéticos, donde se reconoce la legitimidad de una pluralidad de interpretaciones contrapuestas y donde los criterios de validez no son la verdad y la falsedad, sino la plausibilidad y la capacidad de revelarnos su objeto.

El concepto mismo de identidad cultural es problemático. Presupone una homogeneidad y estabilidad cultural que, en los hechos, es cada vez menos frecuente en el contexto de la modernidad tardía. Ante la profundidad y velocidad de los cambios culturales, los nuevos procesos de fragmentación social y la transformación e, inclusive, la desaparición de valiosas tradiciones y mundos de vida, el concepto de identidad cultural aparece, para varios autores, insuficiente para poder captar la diversidad, provisoriedad y diferenciación que se está produciendo actualmente (García de la Huerta, 1999: 11 y 243).

Resulta entonces plausible lo sostenido por Stuart Hall en cuanto a que dicho concepto es "el lugar [mismo] de la dificultad" (Hall, 1997), o lo señalado por Bauman de que es intrínsecamente polémico: toda afirmación identitaria se da en el marco de una lucha con otras identidades, de modo que "las batallas de identidad no pueden cumplir su función de identificación sin dividir tanto o más de lo que unen” (2004: 165-169). Hace tres décadas, Lévi-Strauss había advertido, al respecto: "Se ha comprobado que el contenido de la noción de identidad se ha puesto en tela de juicio e incluso que lo convierte en objeto de una crítica muy rigurosa" (1977: 9).

Frente a esto, algunos se preguntan por qué no abandonar este concepto, como ya lo habían sugerido importantes filósofos y científicos sociales mucho antes de la oleada postmodernista. Para Jean Paul Sartre, el ser humano es "proyecto", una existencia siempre abierta e incompleta, que sólo puede llegar a ser estable con la muerte, que representa, a la vez el triunfo de la mirada de los otros y, paradojalmente, la imposibilidad de autodefinirme (Sartre, 2006 [1943]). Sin embargo, el precio de evitar la equivocidad y las dificultades de su empleo es demasiado alto. No sólo resulta muy difícil encontrar otra categoría que pudiera sustituirla, como reconoce Stuart Hall (1997); sino que, pese a todas sus dificultades, sigue siendo necesario e insustituible. Nuestra opción teórica sería intentar contribuir a su redefinición, complejización, enriquecimiento y flexibilización, más que proponer su eliminación o sustitución por otro término. 
Habría que reconocer que las identidades latinoamericanas son cambiantes, heterogéneas e internamente conflictivas. Como dijimos, el problema de las identidades es siempre plural y lo es en todos los sentidos, no sólo porque toda identidad se constituye en relación con otras, surge y se desarrolla en un plexo identitario, sino porque cada una de ellas contiene una complejidad interna; cada identidad es en sí misma un compuesto con diferentes niveles. De manera que, como ha sucedido con otras relevantes categorías de las ciencias sociales, nuestra visión ha pasado de lo aparentemente simple a algo cada vez más complejo. Si uno revisa trabajos de treinta o cuarenta años atrás, la identidad y los procesos identitarios parecían algo mucho más sencillo de interpretar. Ahora estamos penetrando hacia adentro, y descubriendo cada vez más las dificultades del análisis de las construcciones identitarias.

El examen de las distintas interpretaciones permite también constatar en los textos una tendencia dogmática, al monólogo y de ausencia de criticidad. Los autores, en general, no se refieren a otros, ya sea que sus posturas sean similares u opuestas. Podría decirse que hay un debate encubierto, que no se explicita, y esto exige del lector un esfuerzo intelectual considerable para orientarse y entender lo que diferencia y aproxima las diversas lecturas o "tesis", como las hemos llamado.

Finalmente, puede señalarse que en América Latina y en Chile la preocupación predominante de las ciencias sociales han sido las identidades nacionales, pero ellas siempre han convivido con otras identidades culturales, como las regionales o las étnicas, a las que se les prestaba mucha menor atención. Hoy día parece más importante analizar estas múltiples identidades que, para sus portadores, tienden a ser las más significativas. Reconocer esta trama identitaria diversa nos parece una condición básica para salir de los laberintos de las interpretaciones clásicas de la identidad cultural latinoamericana.

\section{REFERENCIAS}

Arguedas, J. M. (1981). Formación de una cultura nacional indoamericana, $4^{\text {a }}$ edición. Ciudad de México, México: Editorial Siglo XXI.

Bauman, Z. (1999). La globalización. Consecuencias humanas. Buenos Aires, Argentina: Editorial FCE.

----- (2004). Identidad. Buenos Aires, Argentina: Editorial Losada.

Brünner, J. J. (1993). Cartografías de la modernidad. Santiago, Chile: Editorial Dolmen. 
Castells, M. (2006). Globalización, desarrollo y democracia: Chile en el contexto mundial. Santiago, Chile: Editorial FCE.

Cornejo-Polar, A. (1990). "Nuevas reflexiones sobre la crítica latinoamericana", en De Cervantes a Orovilca. Homenaje a Jean-Paul Borel. Madrid: Editorial Viso, 225-235.

Eyzaguirre, J. (1946). Hispanoamérica del dolor y otros estudios. Madrid, España: Ediciones Cultura Hispánica.

------ (1961). Chile en el tiempo. Santiago, Chile: Ediciones Nueva Universidad, Universidad Católica de Chile.

García Canclini, N. (1990). Culturas híbridas. Ciudad de México, México: Editorial Grijalbo-Consejo Nacional de la Cultura y las Artes (CNCA).

----- (2000). La globalización imaginada. Ciudad de México, México: Editorial Paidós.

García de la Huerta, M. (1999). Reflexiones americanas. Ensayos de intra-historia. Santiago, Chile: Ediciones Lom.

Gisbert, Teresa (1999). El paraíso de los pájaros parlantes. La imagen del otro en la cultura andina. La Paz, Bolivia: Plural Editores.

Guadarrama, P. (1992). "Pensamiento filosófico e identidad cultural latinoamericana”. En H. Dieterich (Coord.), Nuestra América frente al V Centenario. Santiago, Chile: Ediciones Lar, 99-121.

Hall, S. (1990). "Cultural identity and diaspora". En Rutherford, J. (Ed.), Community, culture, difference. Londres, United Kingdom: Lawrence and Wishart, 222- 237.

----- (1992). “New ethnicities”. En D. James y A. Rattansi (Eds.), “Race”, culture and difference. Londres, United Kingdom: Sage Publications \& Open University Press, 252-258.

----- (1997). “Introduction: who needs identity?”. En S. Hall y P. de Gay (Eds.) "Race", culture and difference. Londres, United Kingdom: Sage Publications, $1-17$.

Jiménez, G. (1993). "Cambios de identidad y cambios de profesión religiosa”. En Bonfil Batalla, G. (Coord.), Nuevas identidades culturales en México. Ciudad de México, México: Consejo Nacional para la Cultura y las Artes (CNCA), 23-54.

------ (1994). “Comunidades primordiales y modernización en México”. En Jiménez, G. y Pozas, R. (Eds.), Modernización e identidades sociales. Ciudad de México, México: UNAM, 154-169.

Larraín, J. (2000) [1996]. Modernidad, razón e identidad en América Latina, 2da. ed., Santiago, Chile: Ed. Andrés Bello.

----- (2001). Identidad chilena. Santiago, Chile: Lom Ediciones.

Lévi-Strauss, C. (1977). "Prólogo". En La identidad. Madrid, España: Ediciones Petrel, 7-10.

Maffesoli, M. (2004). “Tribalisme postmoderne. De l' identité aux l' identifications". En Infoamérica.org http://1libertaire.free.fr/Maffesoli04.html. (Visitado el 11.11.2009). 
Montecino, S. (2005). "Mestizaje". En Pensamiento crítico latinoamericano. Conceptos fundamentales, tomo II, Santiago, Chile: Ediciones Universidad Católica Silva Henríquez.

Morandé, P. (1984). Cultura y modernización en América Latina. Madrid, España: Encuentro Ediciones, 1987.

Paz, O. (2004a) [1959]. "El laberinto de la soledad". En El laberinto de la soledad/Postdata/Vuelta a El laberinto de la soledad. Ciudad de México, México: FCE, 7-231.

------ (2004b) [1970]. “Postdata”. En El laberinto de la soledad/Postdata/Vuelta a El laberinto de la soledad. Ciudad de México, México: FCE, 233-318.

Pietri, A. U. (1991). La creación del nuevo mundo. Caracas, Venezuela: Grijalbo.

Reynaga, R. (Wankar) (1981) [1978]. Tawantinsuyu. 500 años de resistencia quechua aymara. Ciudad de México, México: Editorial Nueva Imagen.

Rouquié, A. (1997) [1984]. Extremo Occidente. Introducción a la América Latina. Buenos Aires, Argentina: Emecé Editores.

Sartre, J. P. (2006) [1943]. El ser y la nada. Buenos Aires, Argentina: Editorial Losada.

Vergara, J. I. y Vergara Estévez, J. (1996). "La identidad cultural latinoamericana”. Persona y Sociedad Vol. X, No 1, 77-95.

Zea, L. (1993) [1983]. "América Latina: largo viaje hacia sí misma”. En Zea, L. (Comp.), Fuentes de la cultura latinoamericana, Tomo I. Ciudad de México, México: Editorial FCE. 